Article

\title{
A Study Regarding the Representation of the Sun in Young Children's Spontaneous Drawings
}

\author{
José Domingo Villarroel * and Xabier Villanueva \\ Faculty of Education Bilbao, University of the Basque Country, UPV/EHU, Barrio Sarriena, s/n, \\ Leioa 48940, Bizkaia, Spain; xvillanueva001@ikasle.ehu.eus \\ * Correspondence: txomin.villarroel@ehu.eus; Tel.: +34-946-01-7503
}

Received: 29 July 2017; Accepted: 17 August 2017; Published: 22 August 2017

\begin{abstract}
Drawing has historically been the preeminent way of portraying the observations of the sun. The study of the early stages of the development of astronomical thought and the examination of human graphic expression indicate this. With that in mind, it is interesting to note that young children very frequently draw the sun in their spontaneous depictions and, also, that there are preliminary indications that this fact might be related to their conceptual development. This study examines 279 pictures that children aged 4 to 8 spontaneously depicted, paying particular attention to their solar representations and the relationship that they have with other pictorial elements. The data is also related to children's understanding of the inanimate nature of the sun. The results lend weight to the assumption that children do not draw the sun without intent and allow for adding fresh data to the growing body of research showing the importance of considering young children's graphical expression when it comes to gaining insight into their understanding regarding natural phenomena.
\end{abstract}

Keywords: early education; astronomical thinking; natural phenomena; children's drawings; science education

\section{Theoretical Framework}

Drawing has historically been the primordial method to reflect the observations of the sun. Representative examples of this fact may be found along the pathway of astronomical science, particularly, in its pre-photographic times (Vaquero and Vázquez 2009). Some significant cases are, to name but a few, the pioneering solar drawings that the Benedictine monk John of Worcester depicted in 1128 (Lawrence-Mathers 2013) and the Christopher Scheiner's and Galileo Galilei's depictions of the solar sunspots (Hughes 2012).

Visual art also witnesses human interest in solar observations. This is especially true from the seventeenth century on, when the sun, the sky and atmospheric phenomena became the subject of particular attention to landscape painters (Thornes 2000). This also applies to the artists who express a significant interest in eclipses (Pasachoff and Olson 2014) and to the painters strongly influenced by the astronomical discoveries of their time as well (Leach 2016).

The truth is that the sun has been a significant leitmotiv for human pictorial expression long before the initial stages of modern science, perhaps due to the fact that almost every culture has developed theories regarding the movement of the Sun, Earth and planets (Forrester 2013). Ethnoastronomic and archaeoastronomic studies reveal that the representation of the sun, along with other heavenly bodies, has accompanied human graphical expression from even the prehistoric times (Sarhaddi 2013) so that solar depictions are "the most favored and easiest to be recognized on archaeological artifacts" (Pásztor 2015).

Interestingly, young children also draw the sun (Dimitrijević et al. 2016; Konyushkova et al. 2014; Ulker 2012; Yilmaz et al. 2012). More importantly, previous research shows that when they are given a free hand, the sun turns out to be a recurrent pictorial element appearing in their drawings (Ahi 2017; Akerson et al. 2015; Anderson et al. 2014; Sanz 2015; Villarroel et al. 2017). 
One might reflect upon what motivates children to freely represent the sun and whether this fact might have any connection with their cognitive development. In this respect, there is preliminary evidence to suggest that children under the age of eight do not draw the sun accidentally and that this pictorial tendency might be related to their process of grasping natural phenomena (Ahi 2017; Anderson et al. 2014; Villarroel 2016; Villarroel and Ros 2013; Villarroel and Infante 2014). These initial indications would certainly be consistent with the current consensus with the belief that while drawing, young children express their thoughts and feelings linked to their daily life experiences (Beauregard et al. 2017; Steyn and Moen 2017). This belief sparks methodological approaches based on the examination of young children's drawings with a view to gaining insight into their conceptual development (Alford 2015; Bartoszeck and Tunnicliffe 2017; Izadkhah and Gibbs 2015; Villarroel and Sanz 2017).

Nevertheless, no research has explicitly intended to examine the representations of the sun in early childhood and, more specifically, whether children display their solar depiction in accordance with any pictorial pattern or whether this pictorial tendency has any connection with their conceptual development. In this regard, young children tend to consider the sun as a living thing and, also, they attach anthropomorphic features to it (Kallery 2011) but no previous study has covered the possible connections between these beliefs and young children's graphical expression.

Conforming to this theoretical framework, this research project poses the question of to what extent young children's pictorial tendency to represent the sun should also be considered as an early manifestation of their cognitive awareness linked to sky observations.

To this objective, the present study aims to carry out an in-depth examination of the spontaneous representations of the sun appearing in young children's drawings and more specifically it pursues the following key objectives:

1. To study the pictorial content of the drawings that children between 4 and 8 years of age freely carry out on a generic topic linked to the environment, with particular focus on the following issues: whether or not the sun is drawn, the pictorial characteristics (size, colour and position) through which the sun is displayed and the relation that this particular drawing presents with other pictorial elements.

2. To examine whether the pictorial variables posed in the previous point differ in relation to both the educational level of the children comprised in the sample and the gender variable.

3. To inquire into the understanding that the children in the sample express regarding the concept of living things by examining their responses to a categorization task, paying special attention to their answers concerning the sun and connecting their pattern of responses with the pictorial characteristics of the drawings of the sun.

Ultimately, this research intends to provide new evidence concerning the liaison that graphical expressivity in early childhood has with children's comprehension of natural phenomena.

\section{Method}

\subsection{Sample}

The data under study comes from interviews made with 279 children (156 girls and 123 boys). Eighty-two of these interviews were undertaken in 2012 and 2014 within a more general research project aimed at studying the early understanding of plant life. The remaining 197 interviews were carried out in 2015. All the interviews comprising this study were conducted by the signatures of the present paper in strict accordance with the methodology described in the section of data collection.

At the time when the data under study were gathered, the children participants were enrolled in either the 2nd cycle of Pre-primary Education or the 1st cycle of Primary Education. Table 1 details the composition of the sample regarding the academic year and age of the children in the sample.

Five medium-sized state-run-schools were visited in turn by the researchers to collect the data. The selection of the schools was made under the criterion of proximity and accessibility to the members of the research team and all of them are based in the province of Biscay (Basque Country, Spain). 
Table 1. Age and academic year distribution of the children comprising the sample at the time when the interviews to the participants were made $(N=279)$.

\begin{tabular}{cccc}
\hline Educational Stage & Academic Year & Age & $\mathbf{\%}$ \\
\hline \multirow{2}{*}{ Pre-primary Education } & Penultimate year & $4-5$ & 22.9 \\
& Final year & $5-6$ & 30.1 \\
\hline \multirow{2}{*}{ Primary Education } & First year & $6-7$ & 26.9 \\
& Second year & $7-8$ & 20.1 \\
\hline
\end{tabular}

\subsection{Data Collection}

The data under study comes from two tasks posed to the children during individual meetings. These two activities were, first, an unprompted drawing activity of plants and, second, a verbal questionnaire focused on distinguishing inanimate entities from living things. The following is a more detailed description of the tasks and the procedure used during the individual meetings.

Regarding the pictorial task, the children were proposed to accomplish a free depiction on the general topic of plant life. With a view to encouraging the children to engage in the pictorial activity, the task was presented in the classroom by one researcher some days before the beginning of the activity. As for the presentation involving the younger children in the sample (those in Pre-primary Education), the researcher introduced a puppet he brought with him and told a story about the little that the puppet knew about plants. The children were encouraged to help the puppet understand what plants are by means of a drawing. Furthermore, in the case of the presentation of the activity to the older children (Primary Education), they were prompted to undertake a drawing of plants in order to express their understanding on the topic but no story was told to them.

Concerning the structure of the interview, firstly, a blank piece of paper and a pencil were given to the child and they were reminded that the goal of the activity was to draw a picture about plants. No additional clue concerning possible pictorial elements to be drawn was given to the children and they were allowed a free hand to tackle the activity. More specifically, no particular indication was offered in connection with the depiction of the sun or other elements appearing in the sky.

After the child indicated that the picture was finished, the researcher asked them about the meaning of all the pictorial elements displayed by the child. The researcher took note of the meaning expressed on each of the issues drawn. Subsequently, ten coloured markers were put at the child's disposal and it was suggested that the picture could be coloured. Again no additional indication was given concerning the colours to be used to colour the drawn elements. The ten colours were displayed in random distribution and in all the cases were as follows: violet, gray, orange, blue, pink, yellow, black, green, brown and red. Similar procedures concerning pictorial tasks have been undertaken in previous research (Sanz 2015; Savva 2014; Villarroel and Ros 2013).

Once the colouring activity was finished, the researcher initiated the second task of the meeting related to the assessment of children's ability to correctly differentiate inanimate entities and living beings. This test was designed in accordance with the proposal of Leddon et al. (2009) as follows: eight photos showing close-ups of different entities (18 cm long and $10 \mathrm{~cm}$ wide) were presented one by one to the child. The entities displayed were the sun, some clouds, a motorbike, a vehicle, a tree, a dog, two flowers and a bird.

At the beginning of the test and with the objective of being sure that the child did not have any doubt about what the entity appearing in the photo was, the researcher asked the child about what was displayed in the picture. In no case did the children find it difficult to recognise the entities shown in the photographs. Then, the child was asked to consider whether the entity appearing in the image was or was not a living being. To that end, the researcher always asked them the same question: 'Is [the entity in the picture] a living thing?' The researcher noted the answers given by the child.

Regarding the place to carry out the interviews, it was agreed in advance with the teacher responsible for the classroom. A separate place inside the classroom, appropriately organised to enable 
the children to be involved in the activity, was mostly the place used to undertake the interview. In some cases and at the teacher's request, the meeting with the children was made outside the classroom but close to it in order to prevent the children from feeling uncomfortable.

As for ethical procedures, the board of directors of the schools participating in the study and the teachers whose classroom were involved in the study were informed in advance concerning the objectives and procedures of the research projects. Families of the children were also informed in writing with regard to the methodology. Permission in writing to carry out the study from both directors of the schools and the families of the children were requested. The research procedure was monitored by the Ethic Committee for Researching with Human Beings at the University of the Basque Country (CEISH/214/2013/Villarroel Villamor)

\subsection{Variables and Statistical Procedures}

The variables considered for the study include the following:

1. Sex, age and academic year of the children.

2. Regarding the depiction of the sun:

a. Whether or not the child had drawn the sun. This is a categorical variable and takes one of two possible values, the representation appears or it does not appear.

b. The list of pictorial elements displayed above the land or the ground level in the picture (for instance, the Moon, stars, the sky, a rainbow, and the representation of atmospheric phenomena, such as rainfall, clouds and so on). This is a categorical variable for each of the pictorial elements and takes one of two possible values, the representation appears or it does not appear.

c. The colour chosen to represent the sun and the total area coloured. To specify the area coloured, the drawings were, firstly, digitalised and then the area covered by the sun was measured by means of the software ImageJ http://rsb.info.nih.gov/ij/ (Schneider et al. 2012). This is a continuous variable.

3. Regarding the test linked to the study of children's ability to accurately classify inanimate entities and living beings, the variables under study include the following:

a. The number of incorrect responses to the question of whether each of the eight entities displayed was a living being. This ordinal variable ranges from 0 to 8 .

b. Whether or not the sun had been wrongly classified as a living being. This is a categorical variable and takes one of two possible values, right or wrong.

Regarding the statistical procedures, the liaison between categorical variables was studied by Chi-square and the effect size by means of the Cramer's V. Moreover, the study of gender differences was undertaken by the Mann-Whitney test and, in this case, the Pearson's correlation coefficient ( $r$ ) was used to measure the effect size. Finally, the Kruskal-Wallis test was chosen to examine the association between discrete and categorical variables and the effect size was estimated throughout the parameter Eta-squared $\left(\eta^{2}\right)$.

\section{Results}

The results of the study are introduced in three sections. The first part provides general data regarding the pictorial elements found in the sample under study and, along with this, the results in connection with the test to evaluate the participants' skills to accurately differentiate inanimate entities and living things. The subsequent section reports specific details on the characteristics of the children's depictions of the sun and the relationship that it displays with other pictorial elements in the pictures. The latter section accounts for the description of the data linked to the representation of human traits in the drawings of the sun. 


\subsection{First Section: General Data}

A total of 1124 pictorial elements were registered in the 279 drawings that comprise the analysed sample; $78.6 \%$ of these elements appeared displayed on ground level and represent plants, animals, landform elements, buildings, machinery and decorative issues. Some examples of the drawings examined are available in the appendix.

More importantly, $21.4 \%$ of the content in the pictures was distinctly drawn above ground level, in the drawing area allocated for the sky. This included the drawing of the following elements: the sun, in 91 drawings; clouds, in 59; the representation of the sky, in 56 pictures; rainfall, in 28 and a rainbow, in 6 drawings.

No differences between girls and boys were noticed when it comes to the frequency of the occurrence in the depiction of in-sky pictorial elements. Nevertheless, the appearance of the sun (Chi-Square $=11[3] ; p<0.05$; Cramer's V = 0.2), clouds (Chi-Square $=10.15[3] ; p<0.05$; Cramer's $\mathrm{V}=0.191$ ) and particularly, the sky (Chi-Square $=29.65[3] ; p<0.001$; Cramer's $\mathrm{V}=0.326$ ) were significantly different in the drawings undertaken by children in different educational levels. Table 2 displays the frequency of the occurrence in drawing the sun, clouds and the sky with each of the educational levels under consideration.

Table 2. Relative frequency (\%) of the depiction of the in-sky pictorial elements within each of the educational levels under study.

\begin{tabular}{cccc}
\hline & The Sun & Clouds & The Sky \\
\hline Penultimate level of preschool education $(N=64)$ & 15.6 & 7.8 & 6.3 \\
Final level of preschool education $(N=84)$ & 36.9 & 22.6 & 16.7 \\
First level in Primary Education $(N=75)$ & 37.3 & 24 & 17.3 \\
Second level in Primary Education $(N=56)$ & 39.3 & 30.4 & 44.6 \\
\hline
\end{tabular}

Moving onto the results that the participants achieved in the test to analyse the children's capacity to distinguish living beings and non-living entities, $14.3 \%(N=40)$ of the children in the sample were able to accurately classify all the entities under examination. None of those who correctly carried out the test were in Preschool Education. More interestingly, virtually half of the children $(N=138)$ classified the sun as a living thing, while two in five expressed that clouds were alive $(N=120)$. There were no differences between girls and boys regarding their consideration of the living or non-living status of the sun but the relationship that this variable has with the educational level is significant (Chi-Square $=12.76[3] ; p<0.001$; Cramer's $\mathrm{V}=0.21$ ). Thus, about half of the younger children expressed their belief that the sun is alive $(45.3 \%$ in the penultimate level of Preschool Education and $65.5 \%$ at the final level of Preschool Education), the level of agreement with this belief drops considerably among the older participants $(42.7 \%$ at the first level in Primary Education and 39.3\% at the second level in Primary Education).

\subsection{Second Section: Specific Data regarding the Depiction of the Sun}

As previously stated, the sun appears represented in $32.6 \%(N=91)$ of the drawings in the sample under study. In all the cases, the children drew it by means of a circular shape appearing in the upper section of the page. Forty-four children drew it in the upper-right-hand corner of the picture; 23, on the left; and 24 drew the sun in the upper-central-part.

Furthermore, most of the 91 children $(83.5 \%)$ coloured the sun in yellow and, to a lesser extent, orange was also a significant option, in this case for $9.9 \%$ of the children. The remaining tiny proportion of the children left the sun without colouring or opted for blue or red.

More importantly, as one considers the drawings of the older children, the representation of the sun occupies a larger area. Table 3 accounts for the total area filled by the depiction of the sun broken down by the educational levels of the children. The differences presented are significant (Kruskal-Wallis H-test $=12.17[3], p<0.01, \eta^{2}=0.13$ ). 
Table 3. Statistical descriptors of the area occupied by the representation of the sun in pictures, split into educational levels.

\begin{tabular}{ccccc}
\hline & N & Median & Mean & SD \\
\hline Penultimate level of preschool education & 10 & 3.45 & 4.98 & 4.39 \\
Final level of preschool education & 31 & 2.21 & 3.6 & 3.39 \\
First level in Primary Education & 27 & 3.2 & 5.6 & 5.31 \\
Second level in Primary Education & 22 & 8.11 & 9.11 & 7.01 \\
\hline
\end{tabular}

Finally, it deserves mentioning that in the pictures under analysis, the depiction of the sun displays no less than an intermediate connection with the representation of the sky and the drawing of clouds. Table 4 details the connexion that the drawing of the sun has with the remaining pictorial elements found in the pictures.

Table 4. The details of the relationship, measured by the Cramer's V, that the drawing of the sun displays with the other pictorial items identified in the pictures comprising the sample.

\begin{tabular}{cccc}
\hline Medium & Medium-Low & Low & Irrelevant \\
\cline { 1 - 3 } Cramer's V $>\mathbf{0 . 3}$ & $\mathbf{0 . 3} \mathbf{>}$ Cramer's V $>\mathbf{0 . 2}$ & Cramer's V $>\mathbf{0 . 2}$ & \\
\hline & & & Vegetables \\
& & Flowers (0.14) & Leaves \\
& Grass (0.29) & Trees (0.16) & Seeds \\
The sky (0.45) & Ground (0.27) & Fruits (0.17) & Roots \\
Clouds (0.46) & Rainfall (0.25) & Insect (0.19) & Mushrooms \\
& Mountains (0.23) & People (0.14) & Animals (vertebrates) \\
& & A rainbow (0.16) & Vehicles \\
& & People (0.14) & Buildings \\
& & & Decorative items \\
\hline
\end{tabular}

\subsection{Third Section: The Anthropomorphic Representation of the Sun}

A significant feature concerning the representation of the sun in the sample under study is related to the fact that $24.2 \%(N=22)$ of the children who depicted the sun drew it with anthropomorphic characters. More particularly, these children drew the sun with human facial features, adding eyes and lips to their drawing and displaying the sun with emotional expressions and a positive mood. Some examples of the representation of the sun with human facial features are presented in Figure 1.

The depiction of the sun with anthropomorphic characters shows an irrelevant connection with both the educational level of the children (Cramer's $\mathrm{V}<0.1$ ) and their gender (Cramer's $<0.1$ ). However, the data of the present study indicate that there is a significant connection between the representation of the solar disk with human facial characteristics and the belief that the sun is actually a living thing (Chi-Square $=8.5[1] ; p<0.01$; Cramer's $\mathrm{V}=0.305$ ). Thus, $81.8 \%$ of those who drew an anthropometric sun $(N=22)$ expressed the belief that the sun is a living thing, while the proportion drops to $46.4 \%$ in the case of those who drew a sun without any human facial features $(N=69)$.

It is worth noting that the anthropomorphic representation of the sun displays no connection with the classification of the other entities involved in the test; that is to say, with the correct classification of clouds and vehicles, as non-living entities, and plants and animals, as living things. 

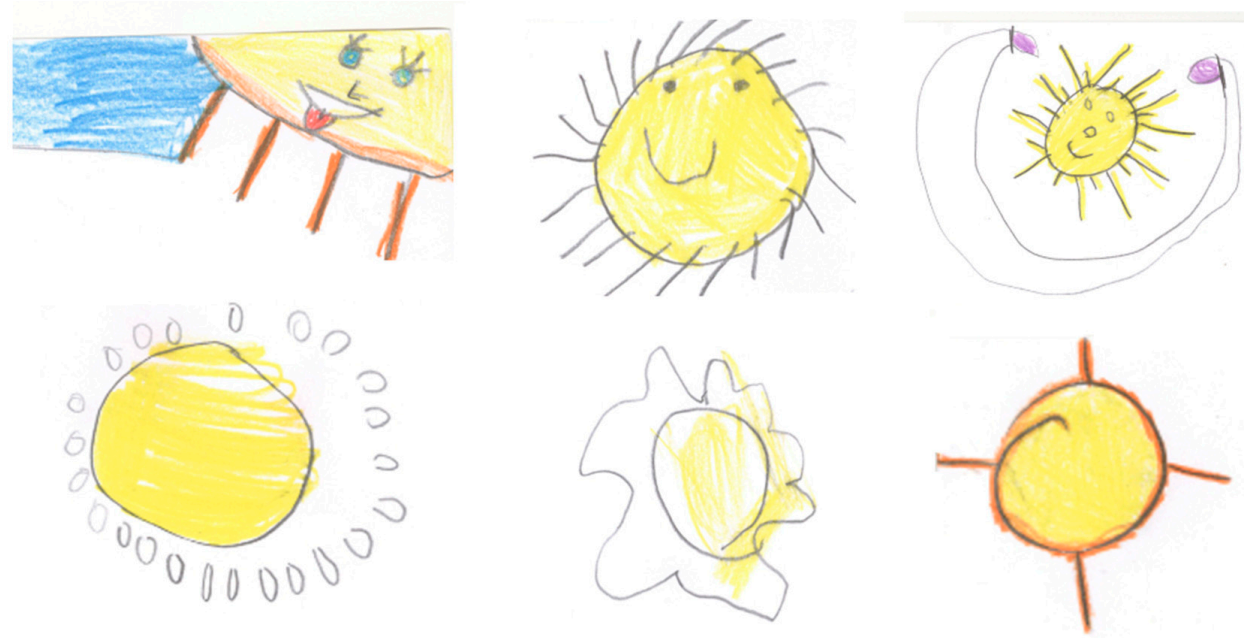

Figure 1. Some examples of the anthropomorphic and non-anthropomorphic representations of the sun found in the sample under study.

\section{Discussion of the Data and Conclusions}

The data presented in this study point out the fact that a substantial proportion of the children in the sample spontaneously drew the sun (slightly more than three in ten) and, also, that this proportion differs in accordance with their educational level. Thus, more than four out of ten children in their second year of Primary Education (7-8) represented the sun, while a little more than one out of ten of those in their penultimate year in Preschool Education (4-5) included it. These observations are consistent with previous research involving the examination of the free depictions undertaken by children at similar educational levels, in the sense that, firstly, the pictorial occurrence of the sun never drops below $30 \%$ of the pictures under consideration and, secondly, the older children stand out for their tendency to carry out solar representations more often (Villarroel and Infante 2014; Villarroel 2016).

More importantly, the present study accounts for the fact that not only did the older children of the sample draw the sun more often than their younger counterparts but they assign larger areas of the picture to represent it. In this connection, it is worth drawing attention to the fact that when it comes to analysing young children's drawings, the size of the elements drawn is not a trivial issue and this pictorial feature is supposed to be associated with the perceived importance and with the significance that the child attaches to the subject matter (Gernhardt et al. 2015; Slee and Skrzypiec 2016). As for the implications that these ideas have in the sample under study, they suggest that the significant relevance that the older children attribute to the sun is the presumable reason which inspires them to draw bigger solar representations.

The data presented also indicates that the occurrence of the depiction of the sun correlates with the drawing of flowers, trees and fruit and shows a medium-low link with the representation of grass. Former research also proves the connection that the representation of plant-related pictorial elements have with the illustrations of the sun; a fact that is understood as a consequence of the process of grasping the role that the sun plays in plant life (Ahi 2017; Villarroel 2016; Villarroel and Infante 2014). However, this study highlights the strong liaison that the depiction of the sun displays with the representation of the sky, with the drawing of clouds and, to a lesser extent, with the pictures of ground, rainfall and mountains.

A final salient finding that this study discloses is the connection found between the anthropomorphic representations of the sun and the lack of understanding regarding its inanimate nature. Thus, almost a quarter of the children who drew the sun, depicted it with human facial features and the study reveals that these children were more prone to consider that the sun is a living thing. Previous research has also confirmed the link between attributing anthropomorphic features to the sun during childhood 
and the inadequate consideration of it as a living thing (Kallery 2011) but, to the best of our knowledge, so far no study has shown that young children's graphical expression may reflect this connection.

To sum up, the evidence collected in this study indicates that the occurrence of the spontaneous representations of the sun in the sample examined means more than an accidental pictorial artefact. Firstly, it follows an age-related pattern, so that the older children are more inclined to display solar representations. Secondly, the older children are also more prone to draw larger representations of the sun which might be an indicator of the extra significance that they attach to this drawing. Thirdly, the sun is predominantly illustrated in tandem with other in-sky pictorial elements and, finally, the anthropomorphic illustrations of the sun seem to be connected with young children's lack of understanding regarding the inanimate nature of the sun.

These conclusions are consistent with the assumption that at some point between the final stage of Early Education and the first courses of Primary Education, young children begin to attach a special significance to the far-off phenomena occurring in that imprecise space commonly called the sky and this would be reflected in their spontaneous graphical expression. This fact might be at the root of the age-related pattern found in this study and it might also be coherent with the aforementioned predisposition that the older children show to spontaneously draw the sun in close connection with the representation of the sky and familiar atmospheric phenomena.

The ancient iconographic linked to celestial objects is understood as the most basic status of the human sky-watching activity and one of the earliest manifestations of human cognitive awareness (Murray 2015). In light of the reflections presented, one might consider that the former is also applicable to young children's artistic activity so that the pictorial patterns observed in this study might be a consequence of the initial developments of astronomical thinking in early childhood.

Further longitudinal research examining the changes in young children's graphic expression and particularly, the issue of how the frequency, the type of solar representations and, also, the relationships that these issues have with other pictorial elements, might eventually confirm the evidence collected in this present study.

Author Contributions: All authors contributed equally to this work.

Conflicts of Interest: The authors declare no conflict of interest.

\section{Appendix A}
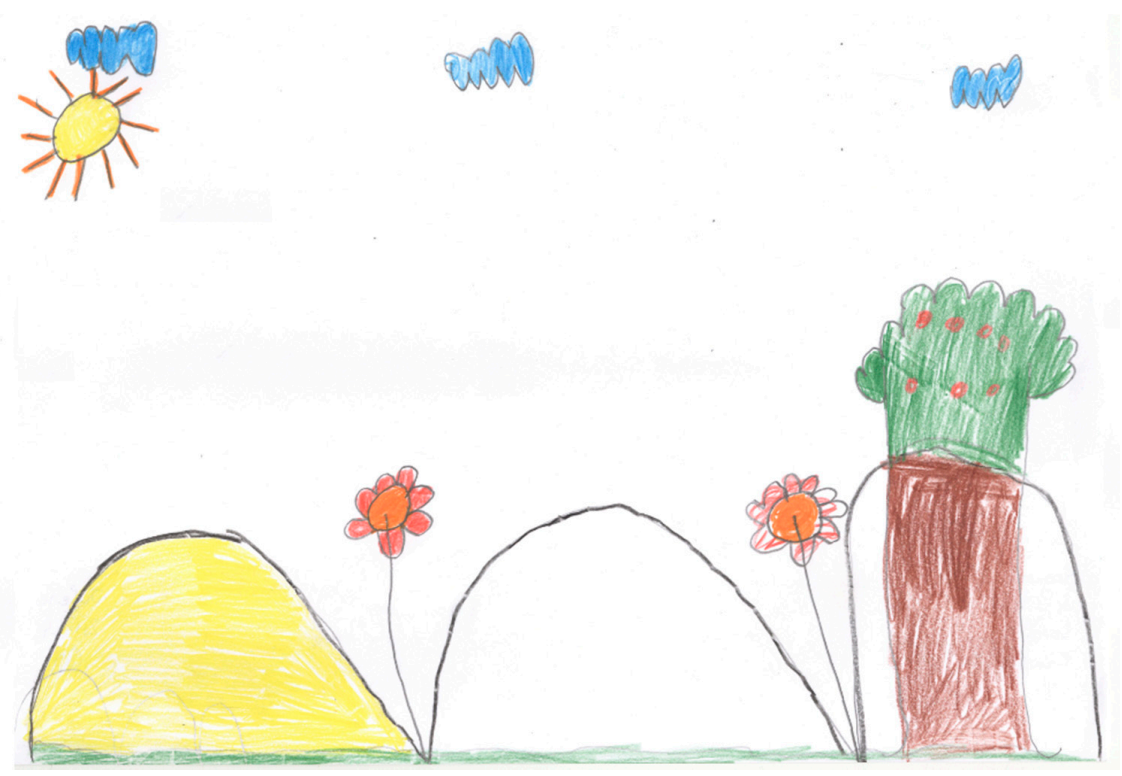

Figure A1. A spontaneous drawing depicted by a 7-year-old child. 


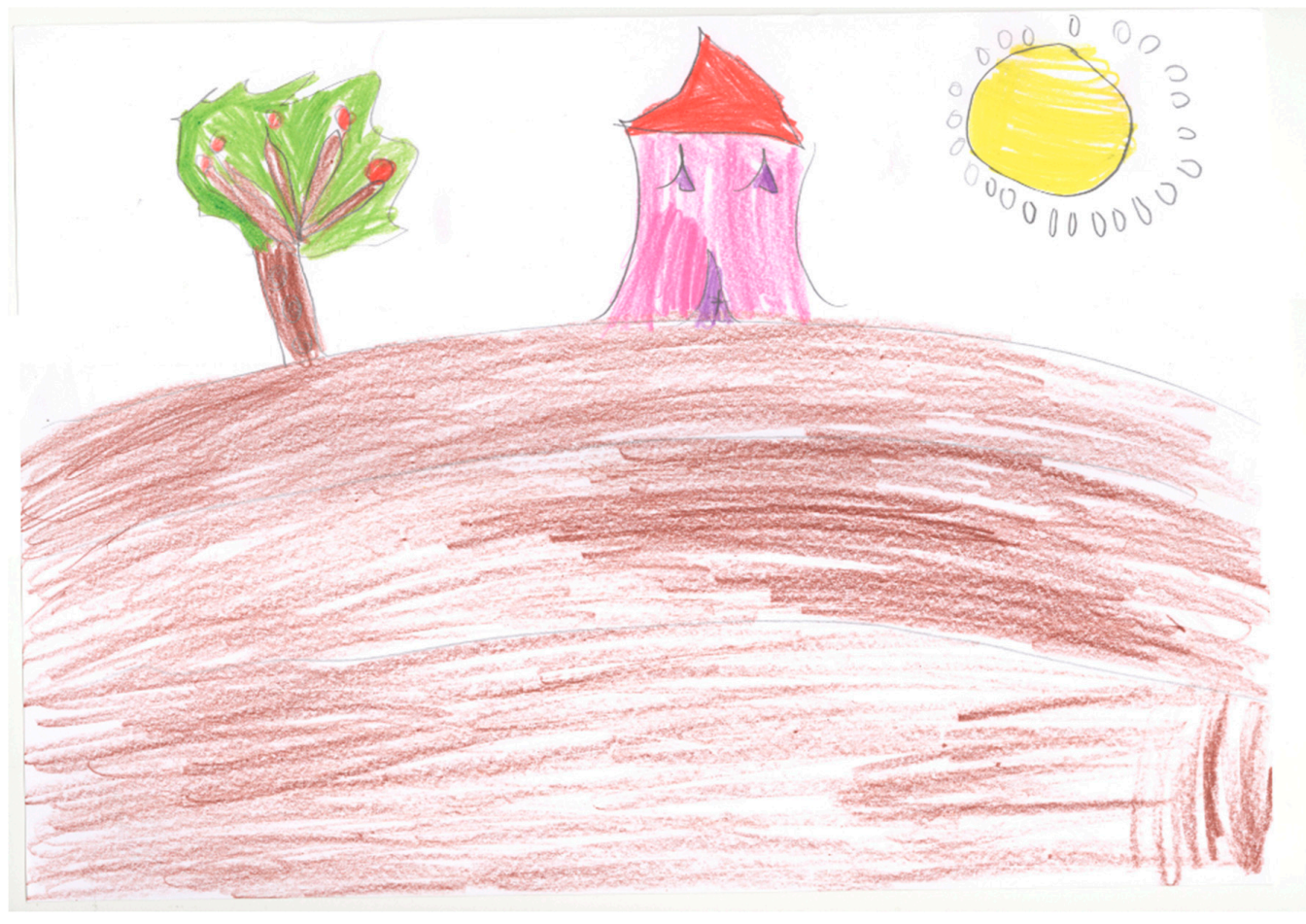

Figure A2. A spontaneous drawing depicted by a 6-year-old child.

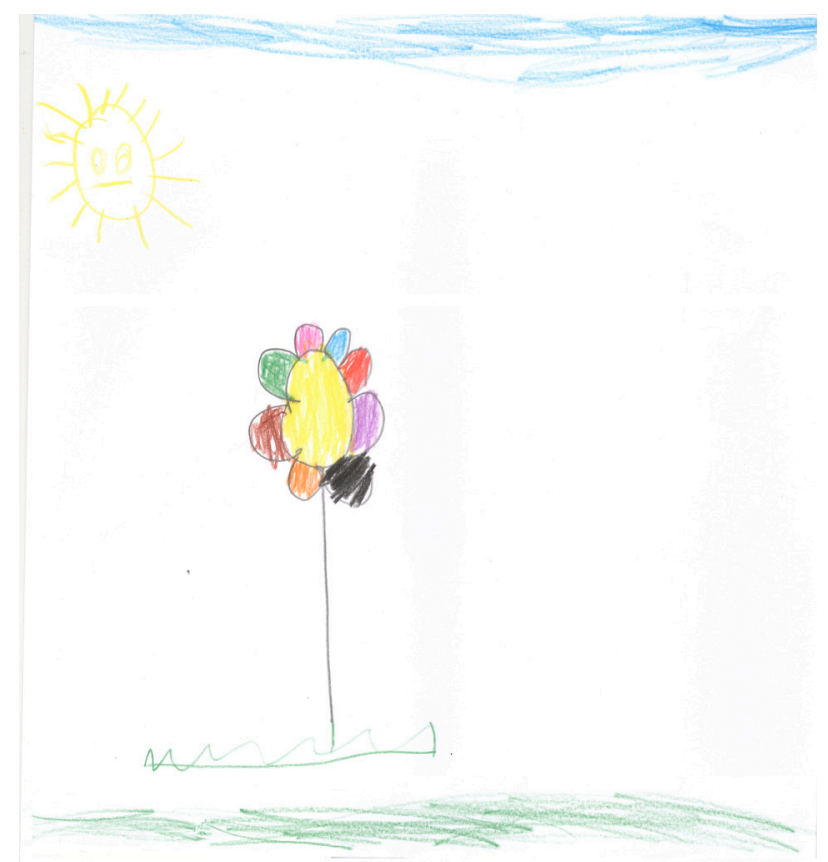

Figure A3. A spontaneous drawing depicted by a 5-year-old child.

\section{References}

Ahi, Berat. 2017. The world of plants in children \textquoterights drawings: Color preferences and the effect of age and gender on these preferences. Journal of Baltic Science Education 16: 32-42.

Akerson, Valarie L., Ingrid Weiland, and Khadija E. Fouad. 2015. Children's ideas about life science concepts. In Research in Early Childhood Science Education. Edited by Kathy Cabe Trundle and Mesut Saçkes. Dordrecht: 
Springer Netherlands, pp. 99-123. Available online: http://dx.doi.org/10.1007/978-94-017-9505-0_5 (accessed on 5 May 2017).

Alford, Christine. 2015. Drawing. The universal language of children. New Zealand Journal of Teachers' Work 12: $45-62$.

Anderson, Janice L., Jane P. Ellis, and Alan M. Jones. 2014. Understanding early elementary children's conceptual knowledge of plant structure and function through drawings. CBE Life Sciences Education 13: 375-86. [CrossRef] [PubMed]

Bartoszeck, Amauri Betini, and Sue Dale Tunnicliffe. 2017. Development of biological literacy through drawing organisms. In Drawing for Science Education: An International Perspective. Edited by Phyllis Katz. Rotterdam: Sense Publishers, pp. 55-65. Available online: http:/ /dx.doi.org/10.1007/978-94-6300-875-4_5 (accessed on 5 May 2017).

Beauregard, Caroline, Garine Papazian-Zohrabian, and Cécile Rousseau. 2017. Making sense of collective identity and trauma through drawing: The case study of a Palestinian refugee student. Intercultural Education 28: 113-30. [CrossRef]

Dimitrijević, Jelena D., Sanja Filipović, and Jelena D. Stanisavljević. 2016. An analysis of students' drawings for the purpose of considering the efficiency of teamwork (programme content: Marine life community). Journal of Subject Didactics 1: 25-38.

Forrester, Rochelle. 2013. How Change Happens: A Theory of Philosophy of History, Social Change and Cultural Evolution. Available online: http:/ / www.rxiv.org/pdf/1502.0237v1.pdf (accessed on 19 June 2017).

Gernhardt, Ariane, Hartmut Rübeling, and Heidi Keller. 2015. Cultural perspectives on children's tadpole drawings: At the interface between representation and production. Frontiers in Psychology 6: 812. Available online: http:/ / www.ncbi.nlm.nih.gov/pmc/articles/PMC4469825/ (accessed on 9 May 2017). [CrossRef] [PubMed]

Hughes, Stefan. 2012. Catchers of the Light: The Forgotten Lives of the Men and Women Who First Photographed the Heavens. Paphos: ArtDeCiel Publishing.

Izadkhah, Yasamin O., and Lisa Gibbs. 2015. A study of preschoolers' perceptions of earthquakes through drawing. International Journal of Disaster Risk Reduction 14: 132-39. [CrossRef]

Kallery, Maria. 2011. Astronomical concepts and events awareness for young children. International Journal of Science Education 33: 341-69. [CrossRef]

Konyushkova, Ksenia, Nikolaos Arvanitopoulos, Zhargalma Dandarova Robert, Pierre-Yves Brandt, and Sabine Süsstrunk. 2014. God (s) know (s): Developmental and cross-cultural patterns in children drawings. ACM Journal on Computing and Cultural Heritage 2: 1. [CrossRef]

Lawrence-Mathers, Anne E. 2013. John of worcester and the science of history. Journal of Medieval History 39: 255-74. [CrossRef]

Leach, Samuel Hollis. 2016. Scientific Imagining: Studio Based Research into Genre Images of Science and How Art Might Interpret Modern Science. Ph.D. dissertation, College of Design and Social Context, RMIT University, Melbourne, Australia, January.

Leddon, Erin M., Sandra R. Waxman, and Douglas L. Medin. 2009. Unmasking "alive": Children's appreciation of a concept linking all living things. Journal of Cognition and Development 9: 461-73. [CrossRef] [PubMed]

Murray, William Breen. 2015. Astronomy and rock art studies. In Handbook of Archaeoastronomy and Ethnoastronomy. Edited by Clive L. N. Ruggles. New York: Springer, pp. 239-49. Available online: http://dx.doi.org/10. 1007/978-1-4614-6141-8_10 (accessed on 5 May 2017).

Pasachoff, Jay M., and Roberta J. M. Olson. 2014. Astronomy: Art of the eclipse. Nature 508: 314-15. [CrossRef]

Pásztor, Emília. 2015. Celestial symbolism in central european later prehistory-Case studies from the bronze age carpathian basin. In Handbook of Archaeoastronomy and Ethnoastronomy. Edited by Clive L. N. Ruggles. New York: Springer, pp. 1337-48. Available online: http://dx.doi.org/10.1007/978-1-4614-6141-8_129 (accessed on 5 May 2017).

Sanz, Olga. 2015. Acercamiento a la comprensión del concepto de ser vivo en educación infantil. Ikastorratza. e-Revista de Didáctica 15: 99-118.

Sarhaddi, Fereshteh. 2013. Pictograph and petroglyphs of Saravan (Sistan-baluchistan, Iran). Ancient Asia 4: 1-8. Savva, Stavros. 2014. Year 3 to year 5 Children's conceptual understanding of the mechanism of rainfall: A comparative analysis. Ikastorratza. e-Revista de Didáctica 15: 99-118. Available online: http://www. ehu.es/ikastorratza/12_alea/rainfall.pdf (accessed on 1 June 2017). 
Schneider, Caroline A., Wayne S. Rasband, and Kevin W. Eliceiri. 2012. NIH image to ImageJ: 25 years of image analysis. Nature Methods 9: 671-75. [CrossRef] [PubMed]

Slee, Phillip T., and Grace Skrzypiec. 2016. No more bullying: An analysis of primary school children's drawings of school bullying. Educational Psychology 36: 1487-500. [CrossRef]

Steyn, Miemsie, and Melanie Moen. 2017. Drawing sadness: What are young children telling us? Early Child Development and Care (03/23): 1-15. Available online: http:/ / dx.doi.org/10.1080/03004430.2017.1301936 (accessed on 5 May 2017). [CrossRef]

Thornes, John E. 2000. A brief history of weather in European landscape art. Weather 55: 363-75. [CrossRef]

Ulker, Riza. 2012. Turkish children's drawing of nature in a certain way: Range of mountains in the back, the sun, couple of clouds, a river rising from the mountains. Educational Sciences: Theory and Practice 12: 3173-80.

Vaquero, José M., and Manuel Vázquez. 2009. The Sun Recorded through History: SCIENTIFIC Data Extracted from Historical Documents, Astrophysics and Space Science Library. Berlin: Springer Science \& Business Media, vol. 361.

Villarroel, José Domingo. 2016. Young Children's drawings of plant life: A study concerning the use of colours and its relationship with age. Journal of Biological Education 50: 41-53. [CrossRef]

Villarroel, José Domingo, Álvaro Antón, Daniel Zuazagoitia, and Teresa Nuño. 2017. Young children's environmental judgement and its relationship with their understanding of the concept of living things. Environmental \& Socio-Economic Studies 5: 1-10.

Villarroel, José Domingo, and Guillermo Infante. 2014. Early understanding of the concept of living things: An examination of young children's drawings of plant life. Journal of Biological Education 48: 119-26. [CrossRef]

Villarroel, José Domingo, and Olga Sanz. 2017. A study regarding the spontaneous use of geometric shapes in young children's drawings. Educational Studies in Mathematics 94: 85-95. [CrossRef]

Villarroel, José Domingo, and Iker Ros. 2013. Young Children's conceptions of rainfall: A study of their oral and pictorial explanations. International Education Studies 6: 1. [CrossRef]

Yilmaz, Zuhal, Milan Kubiatko, and Hatice Topal. 2012. Czech children's drawing of nature. Educational Sciences: Theory and Practice 12: 3111-19.

(C) 2017 by the authors. Licensee MDPI, Basel, Switzerland. This article is an open access article distributed under the terms and conditions of the Creative Commons Attribution (CC BY) license (http:/ / creativecommons.org/licenses/by/4.0/). 